\title{
H-PSO: A SECURE ROUTE OPTIMIZATION MODEL FOR LINK FAULT DETECTION IN OPTICAL NETWORKS
}

\author{
J. Kumarnath ${ }^{1}$ and K. Batri ${ }^{2}$ \\ ${ }^{1}$ Department of ECE, PSNA College of Engineering and Technology \\ ${ }^{2}$ Department of ECE, PSNA College of Engineering and Technology
}

\begin{abstract}
In this paper, a new Fault detection and network survivability method is presented based on Enhanced particle swarm optimization (PSO) technique intended for the optical networks. The Heuristic H-PSO algorithm developed from particle Swarm optimization, and it is used for locating the failures position through finding the fitness value of the aforementioned links. The greedy algorithm is used for wavelength assignment. The novelty of this work exists in the form of fitness function that uses bandwidth availability and packet delay as a parameter metrics that ensures the quality of the link paths. As a result, it determines the non-functioned links and the components. This algorithm gives interesting results that verify the logical optics topology that requires to be sustained over the physical network despite of great traffic tenseness. The proposed algorithm is validated considering practical tests with the use of Network Simulator (NS) - 2 by patching OBS in Mesh topology. Results obtained through this optimization model shows promising and superior result with respect to Blocking Probability, End to End delay, Throughput and Burst Loss Rate. Numerical Result shows that there is a decrease in Blocking Probability, End to End delay and Burst Loss Rate in turn shows predominant increase in Throughput compared to E-OBS, E-BSL and E-BSL D methods.
\end{abstract}

Keyword: Failure Detection, Particle Swarm Optimization, blocking probability, Burst loss.

\section{INTRODUCTION}

WDM is developing into a promising technology for ultra-long- haul networks, in which data are traversed in optical channels referred as light-paths. The assigned light path fully utilizes the entire wavelength for a successful transmission in the absence of wavelength conversion technique. The various optical communication network structural design was motivated due to the ultimate improvement in WDM technologies. WDM networks need a fast and cost-effective fault localization and detection mechanism to identify the faults in the network. WDM technologies facilitate the light communication fiber to perform 200 wavelengths functioning at 40 Gbps. Such highly efficient networks are vulnerable to failures that fallouts in the disruption of transmission. The small trouble of network service instigates to serious network fault and it leads to a heavy data loss in fast optical networks. So, it is essential to locate and identify these failures instantaneously. The network failures could be caused by internal or from external sources. The internal factors are network topology, components failure and transmission criteria. The external factors are attacking such like Data Delay, Service Denial and QoS degradation.

Faults are unavoidable in any communication networks. Rapid recognition and quarantine of the fault is indispensable on behalf of the security and reliability of both the network and the amenities conceded over it. [1]In optical networks the attacks may degrade efficient light communication. All non-functions or misleading could not be assumed as attacks. But sometimes the attacks cause network failures in light communications. Attacks may blowout to several users and many locations of the network, whereas a hardware failure only disturbs those connections passing through it. Attacks attempt to elude detection, while failures cannot do that. Rerouting traffic links via an arrangement to endure hardware letdown that could not resolve the difficulties affected by an attack connection. The above said problem is addressed in lot of researches. This research mainly suggested a model to maintain optical monitors for controlling alarms when it finds any single link failure. This monitoring information is succumbed to the control plane of the Optical networks. Through this failure analysis model, all routing information could be achieved regarding the failure and action could be taken for real time traffic restoration.

In order to rectify the issues, many research works were carried out that lies mainly in two categories. [2] Black box model and network model-based methods. The entire network is considered as a black box that

Received JUL 06, 2021

www.dynamicpublishers.com;

www.dynamicpublishers.org;
ISSN1056-2176(Print); ISSN 2693-5295 (online)

$\$ 15.00$ CDynamic Publishers, Inc.

https://doi.org/10.46719/dsa202130.11.01 
involves in the detection of network faults. It generates particular output data when it encounters network failures. The correlation among the network failure and output diagnosis could be obtained over and done with machine learning mechanisms. The advantage of this approach is that there is no need to know the network topology and this method provides proper determination in large scale networks. However, this approach suffers with late learning strategy. Contrast to this black box model, a network model that depends on the network topology is introduced. This model functions based on components and transmission criteria. [3]The fault detection is identified with the throughput of the network. These types of models are well suited for IP networks but suffers with WDM networks with dynamic light path provisioning. The topology of the network model cannot be changed automatically with respect to the dynamic changes. It is time consumable one and it provides improper detection in the strict time constraint environment.

All existing research focused the cost minimization of monitoring reports in failure localization. However [4]dynamic network models suffers in the aspect of both inaccuracy and slow fault localization. To handle these issues, we propose a PSO centered enriched algorithm that could endowment a connection inside a fraction of a second in lieu of fault determination and localization of failures. PSO[28][29] is a population regarding global optimization practice that was developed related with evolutionary algorithms.

This procedure could be practiced practically to elucidate NP-hard optimization process. The furthermost advantage of this algorithm is that the parameters can be adjusted to attain new modified algorithms depended to the associated problems.

In this research effort, we introduce a novel Fault detection and recovery method centered on the swarm algorithm optimization technique. This method is used for fault detection accompanied with the denial attacks that restrict the usage of attaining the particular bandwidth in optical communication. The compensations of this aforementioned system versus the other approaches are dynamic and fast in nature. The presented approach is considered especially to identify network faults caused by denial or inference attacks and the proposed algorithm can be implemented as an enriched algorithm on original PSO. The novelties of this particular work are:

- Particle Swarm Optimization technique is used to identify the fault detection due to the attacks or component failures that restrict the usage of attaining the particular bandwidth in optical communication without using wavelength conversion technique.

- Novel fitness function instantaneously proceeds keen on both the overall wavelength availability and amount of free wavelengths obtainable over the complete path identification. Packet Delay is considered as a significant parameter in determining the failure nodes.

- The proposed fitness function uses the two factors that enforce reliability in the optic fiber. Availed Free wavelength, delay factor are the two primary factors that is used to oversee the impact of hoplength and availability of wavelengths. The fitness value of the aforementioned particle decides it's noteworthy in the path estimation.

The remaining sections of this paper are structured as the following succeed: The Fault detection and security mechanisms used for optical networks are discussed with the aforementioned benefits and detriments in Section II. We present the system architecture and the proposed Detection model in Section III. The experimental outcomes of both existing and proposed fault detection and security mechanisms are analyzed and compared in Section IV. The overall summary and future enhancement of this paper are stated in Section V.

\section{RELATED WORK}

There are two approaches to overcome link failure. The first one is proactive approach that focuses on protection before link failure and the next one is reactive approach that focuses on Restoration. In this section, the issues befall in survivability of optical layer in the optical communication and the methodologies that are used to provide a better solution for physical optical fiber security are surveyed with its advantages and disadvantages. 


\section{Survivability analysis in elastic optical networks}

Survivability plays a key province in recovering from the link and path failures. Li, et al. [6] Studied various issues and confrontations in the link survivability. This work analyzed the impacts of spectrum conversion, configuration, physical layer deficiencies and restrictions, availability and protection path centered spectrum defragmentation in the view of survivability. Different Network protection and restoration schemes were reviewed. Agrawal, et al. [7] Utilized Seismic Zone Information for the recovery of optical networks during the occurrence of natural disorders. The offered node relocation concept entails additional expenditure on fiber link deployment from the original node location to the new node location. Wang, et al. [8] Proposed a new method based on protection scheme that implied the idea of advanced pre-computing and reservation of backup resources such like routes and wavelengths. Distance adaptive dynamic routing and spectrum assignment was implemented using The SBPP algorithm that enhanced the security of light paths. Bhandari and Malhotra [9] Categorized the link faults as Proactive Approach and reactive approach. It emphasized the protection/ restoration keen on link and path of optical fiber. Ghodasara et al [10] suggested the design of the controller in the network management system (NMS) and the control state-machine in the carrier Ethernet switch routers(CESR) and their corresponding interactions. NMS possesses footpath of provisioned bandwidth on every respective CESR port. New service is configured concerning to a precise port on every occasion. This design seams to provide better option than OSPF/CSPF protocols in the optical domain. It focus mainly in providing service in SDN platform. Walkowiak, et al. [11] Applied metaheuristic method to afford close prime clarifications to RSA based Committed Path Protection. Tabu Search algorithm (TS), and a hybrid Adaptive Frequency Assignment-TS (AFA/TS) algorithm is implemented on behalf of directionfinding and spectrum endowment in endured elastic optical networks. TS algorithm be convulsed from weak scalability in greater problematic occurrences.

B. Restoration methods

Chen, et al. [12] Offered an innovative method built on Preplanned FIPP $P_{P-C y c l e s}$ that can support optical WDM network with respect to single Link and dual link failures. This recently proposed technique reduces the restoration time commencing link failure as a result decreasing the time complexity. The keen idea of this approach is backup resource sharing. Giorgetti, et al. [13] Introduced the concept of Traffic Engineering Database that consist of topology related information and spectrum accessibility info. The idea of this work is computing the disrupted light paths via the stored light path database and the resources are freed that was engaged by disrupted light paths. Then backup approach is implemented for every dislocated light path. This approach reduced the recovery time and blocking probability.

Kushwaha and Gumaste et al [14] proposed model based on FISSION architecture for fault tolerant in DC based optical networks. It is a supporting protocol that helps in assisting large DC fabrics. It imposed an interconnection model and

Switchless backplane that support up to million servers. Amar, et al. [15] Used transponder data rate elasticity for optical restoration. It is a costly tactic that confirms unsurpassed exertion traffic recovery irrespectively of network topology and high data rate transponders. Lee and Sriram et al.[16] proposed a new routing strategy known as Contention-Based Limited Deflection Routing (CLDR) scheme in which routing is done dynamically, whether the burst should be resent commencing source or deflection routed . If the assessment leads to deflection route, at that point the same is completed via a path that is focused on the measures like the node configuration, network topology, a set of attributes cope with the node and link paths, and restraints belong to resource boundaries. This research found a prime alternative path lessening a cost function that obviously taken into account for the contention rate as glowing as the burst hop distance. Huang and Yoshida et al.[17] suggested a method intended for Optical Circuits in OPS based Data Center Networks based on Bypass Routing Scheme. A shortest path known as Express path (ExP) is earmarked commencing source en route for destination afore the start of the communication. Then packets of the flow will be routed sideways in the recognized Express path and acquire no loss or contention. After the transmission is done, 
the Express path could be expired. But the proposed idea fails by wavelength reservation degrade the performance when at the time of vast connection establishment.

\section{Optimization Methods}

Hassan and Phillips [18] Proposed a new Particle Swarm optimization algorithm for dynamic RWA problem under the wave length continuity constraint. It employs First-Fit algorithm for wavelength assignment. The novelty of this work lies in the computation of fitness function that used controlled path length and regularized number of free wavelength accessible in the course of route assessment. The performance of this work was witnessed as better when comparing with GA algorithm. The Particle Swarm Optimization (IPSO) algorithm [30] was introduced to classify the most important regions during mobility in $2 \mathrm{~d}$ Video surveillance. Leung and Sing et al.[19] proposed the neural network tactic to reduce the blocking possibility proceeding in optical networks. Optical network parameters were given as input for the neural network layer and the output was to reduce the blocking probability of the optical network. It consider few input parameters of an optical networks 1) APL and CR 2) C and W related to network topology 3) E and D related to optical links, Complex data cannot be stored in buffer less OBS. Bhatt, et al. [20] Proposed a modified ACO algorithm ACOMFL for the fault restoration in OCN. In the first stage it detects the link or path failures through the ants and in the next stage restoration is done with the following ants that determine the alternate path. Harpreet Kaur, et al. [27] Proposed Ant colony optimization methodology to assign the wavelength for data transmission as well as the methodology is implemented to reduce optical Signal to Noise Ratio in NoC. Proposed a modified ACO algorithm for resource minimization and availability of the networks. The novelty of this algorithm lies in a path construction method based on tabu-table technique for formulating achievable paths commencing the source to every single receiver.[5] utilized the movement of the snake for cost matrix formulation so as to optimize the optical network. Pavani, et al. [22] Analyzed the uncertain problems in the implementation of ACO algorithms in optical networks in three categories of byzantine failure such like misdirection of onward ants, plummeting of forward ants and plummeting of backward ants. The corresponding letdowns disturb the routing info of the entire optical network. [25] Improved the effectiveness of the light paths routing and spectrum assignment (RSA) schemes with the evolutionary algorithm. Also balanced the security level by the game theory. From the survey, the major issues in the survivability of optical networks in the occasion of path/link failures are analyzed, and also the intention of the previous works is investigated with its identifiable merits and demerits. A most suitable survivability method should have least network rebuilding time from network failure and the alternative backup hop length should be minimal. Supreme level of former research efforts on single link failures despite the fact that the dual link failures are quite rare. Also, some other research works addressed these issues by introducing efficient mechanisms based on routing and wavelength assignment policies, but it has increased the overhead and computational complexity. To solve the problems that were pointed out on this survey, this paper endeavors to develop a new restoration scheme by implementing population based stochastic optimization technique.

\section{Proposed MeTHOD}

A suitable route is nominated randomly for each connection request from the early determined k-shortest paths. Every associated route is recognized by a distinctive route-id. A common edge table is connected with every vector of route ids. It maps the connection edges in the case of routes passing over an edge in the fiber network. It also assures the information about overloaded edges. In the case of link failure, the neighborhood nodes of the corresponding failed link must aware of the failure and these should be informed to the source and destination nodes of the connections which are passing over that non-functioned link.

\section{A. Particle Swarm Optimization}

\section{Step 1: Initialize the particles.}

The vector of route-id is considered as particles which covers the overall problem space to find best optimal routing solutions. Initialization of this particle could be done in a random manner. The overall population is 
the set of nodes returned with reply from destination. From this corresponding route reply the parameters of the Intermediate Nodes are acquired. The foremost factors that we consider here is Bandwidth and delay.

Step2: The fitness value of every particle is computed using fitness function

The fitness function quantifies fitness value of each particle normally calculated based on multi objective parameter which is used for the optimization in each generation. Every partaking particle has its individual best $p_{\text {best }}$ and the overall best value of all agents $g_{\text {best. }}$. The swarm (Particle) has both velocity and placement position which listen the flying of particle. For every iteration new generation will be created associated with its new velocity and position value. The velocity and related position of agent update equations are specified below as (1) and (2) respectively.

Calculate swarm velocity $V i=V i+c 1(P b i-X i)+$

$c 2(G b i-X i)$

(1)

Where, $(G b i-X i)$ indicates the estimated routes that varies in the point of global best particle and current particle. $(P b i-X i)$ signifies the routes that varies in the point of local best particle and current particle. $\mathrm{C} 1$ and $\mathrm{C} 2$ denotes social learning parameters that finds the faults of the links. It also involves in finding the number of routes to be substituted

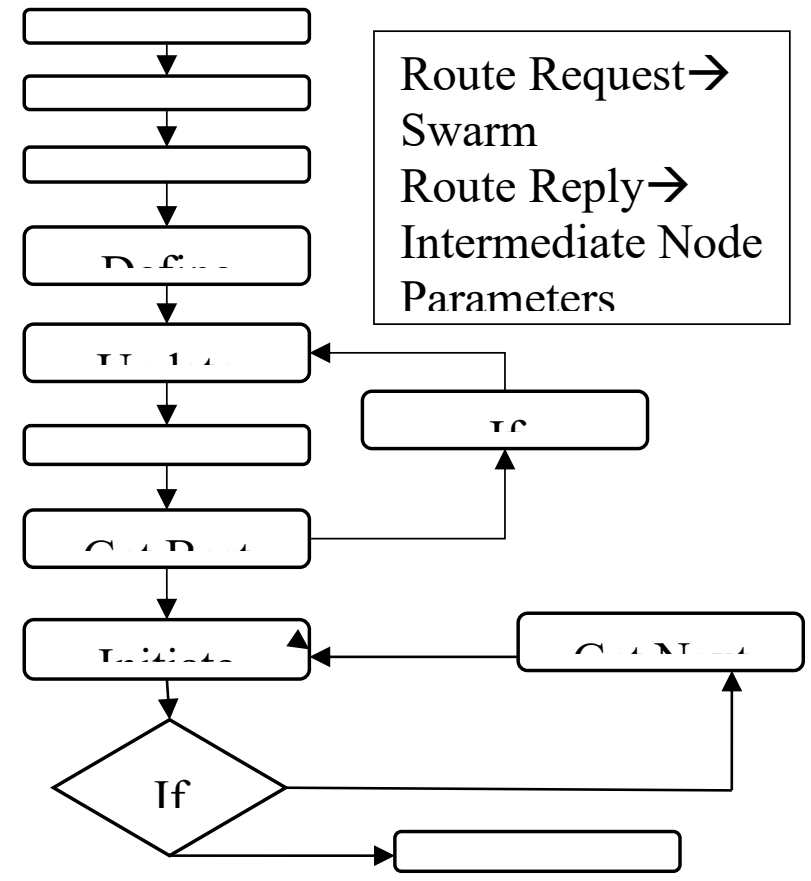

Figure 1. Work Flow of H-PSO

Step 3: The global best value of the particle is taken from the particle which has high fitness value. And the local best particle is observed from the neighbourhood particle that has best fitness value. Update next current position of swarm $X i+1=X i+V i \quad$ (2)

The parameters used in H-PSO algorithm are termed in Table.1.

Table.1 variable description

\begin{tabular}{|c|l|}
\hline Variables & \multicolumn{1}{|c|}{ Description } \\
\hline$V i$ & Velocity of new Particle \\
\hline$P b i$ & Position of individual Particle with best Fitness in $i^{\text {th }}$ hop \\
\hline$X i$ & Current Position in $i^{\text {th }}$ hop \\
\hline$c 1$ & Individual router confidence factor \\
\hline
\end{tabular}




\begin{tabular}{|c|l|}
\hline$c 2$ & Swarm confidence factor are possitive constants \\
\hline$G b i$ & Global Best Fitness in $\mathrm{i}^{\text {th }}$ hop \\
\hline$(\max L(s, d))$ & maximum length of the route among any source and destination pair \\
\hline$L(s, d)$ & The route length between source's' and destination' $\mathrm{d}$ '. \\
\hline$\gamma$ & Design Parameter which is the range of [0,1] \\
\hline$C(p i)$ & Cost lifetime of $i^{t h}$ light path \\
\hline$R h$ & Request holding period \\
\hline$p L i$ & Life time of $i^{t h}$ light path \\
\hline$\Delta t$ & Adjustment constant \\
\hline$H_{i}{ }^{n}$ & Number hops in of $i^{\text {th }}$ light path \\
\hline$\varepsilon$ & Exponential constant factor which is $10^{-5}$ \\
\hline
\end{tabular}

In general, the position and velocity are represented attributes of particles. The three primary factors decide the velocity value of the particle which are: Momentum, Cognitive learning, social learning. Very first the factor momentum supports to transfer the particle in the optical fiber in the direction of the estimated routing path. Router confidence factor employ the searching done by particle themselves over the intermediate hops. This factor combines the changes happened in particle headed for the best position it has rifled in its operation. Swarm confidence factor decides the effect of manipulation that the exploration done by the further participants of the swarm. This aspect combines the search of associated particles that directs to attain the best position whether global best or local best in the complete swarm.

B. Enhanced H-PSO algorithm Equation's and Derivatives

The formula to find velocity and position are included in the below Enhanced PSO algorithm. This algorithm fly over the problem of searching the optimal routing path space and finds the optima path based on fitness value to transmit the data in the optical networks in the case of fault occurrences. It identifies the link faults and path failures through the route reply. In our modified formula the fitness value is calculated with velocity and position of a particle. Here the velocity is represented as Delay of Reply and position of particle is represented as $\mathrm{i}^{\text {th }}$ Hop in path. The fitness function verifies the constraint whether the expected resources are lesser than available resources for the determined new path. If the condition fails, then again the algorithm encounters the new alternate path.

Enhanced H-PSO algorithm:

Velocity of new Particle $(V i)=$ past velocity $V i+\mathrm{c} 1$ (individual best position $\left(P b_{i}\right)$ - current possition $\left.X i\right)$ $+\mathrm{c} 2$ (global best position of all particle $G b$ - current position $X i$ )-

$\mathrm{c} 1$ is individual router confidence factor, $\mathrm{c} 2$ is swarm confidence factor are positive constants

Initialize particles:

$P b_{i}^{n}=\operatorname{Null}($ Position of particle with best fitness), Gb = Null(Global best fitness)

For each swarm $i$ in $\mathrm{G}$

Initialize the $X i, V i$ randomly;

$P b_{i}=X i$

Evaluate swarm fitness values $\{F\{i)\}$

If $\{G b<F(i)\}\{$

$$
G b=F(i) ;
$$

$$
P b_{i}=X i
$$


End if

End For

Hop Count: Hop count is a rough measure of distance between two Nodes.

The fitness function is calculated based on the distance of the nodes and the base station. The distance between the node should be least in order to provide optimal solution and least congestion

Fitness $=$ Distance_Node $(i, j)+$ Distance_Node $(j, B S)$

Calculate Distance based on RERR msg.,

Distance_Node $(n 1, n 2)=\sqrt{\left(X_{-} \text {Pos } 2-X_{-} P o s 1\right)^{2}+\left(Y_{-} P o s 2-Y \_P o s 1\right)^{2}}$

Where $\mathrm{n} 1=\left(\mathrm{X} \_\mathrm{Pos} 1, \mathrm{Y} \_P o s 1\right)$ and n2=(X_Pos2,Y_Pos2)

$\mathrm{i}$ and $\mathrm{j}$ is the parent nodes, BS is a Base Station.

The Neighbor Node is defined as follows:

Neighbor_Node $=\sum_{\mathrm{k}=1}^{\mathrm{N}} \mathrm{Xi}-$ Distance_Node $(\mathrm{k})$

The Throughput is defined as:

$\mathrm{Th}=\sum_{\mathrm{n}=1}^{\mathrm{N}}((\beta(\mathrm{n})) /(\rho(\mathrm{n})))$

Where $\beta$ - No of Packets Received

$\rho$ - Simulation time

$N$ - Number of nodes

Calculate the fitness values of each router using following function where $\gamma-$ design parameter

$$
F(i)=\left[\gamma * \frac{(\max L(s, d)-L(s, d)}{L(s, d)} * T h\right]+\left[(1-\gamma) *\left(1-\frac{\lambda_{\text {total }}-\lambda(s, d)}{\lambda(s, d)}\right) * T h\right]
$$

$c n t=0 ; / /$ Initialization of number of Iteration

While \{count is less than maximum iteration

For each swarm $i \mathrm{G}$

Calculate the swarm velocity $V i=V i+c 1(P b i-X i)+c 2(G b i-X i)$

Update the next current position of swarm $X i+1=X i+V i$

Evaluate the particles fitness values $F(i)$

Update $P b i$

$$
\begin{gathered}
\text { If }\{G b<F(i)\} \\
G b=F(i) \\
P b_{i}^{n}=X i
\end{gathered}
$$

End If

$c n t++$

End While

For each $j$ in light path $l p$

Calculate the delay factor using mean function

$$
\mathrm{pkt}_{\mathrm{i}}^{\mathrm{k}}=\frac{\sum_{\mathrm{t}=1}^{\mathrm{k}} \sum_{\mathrm{j}=1}^{\mathrm{n}}\left(\mathrm{d}_{\mathrm{j}}^{\mathrm{t}+\mathrm{i}}(\mathrm{i})-\mathrm{d}_{\mathrm{j}}^{\mathrm{t}}(\mathrm{i})\right)}{\mathrm{k} * \mathrm{n}}
$$


Delay covariance between two routers $r_{i}, r_{j}$ is

$\sigma\left(\mathrm{r}_{\mathrm{i}}, \mathrm{r}_{\mathrm{j}}\right)=\frac{1}{\mathrm{~K}-1}\left(\sum_{\mathrm{k}=1}^{\mathrm{K}} \mathrm{pkt}_{\mathrm{i}}^{\mathrm{k}} \cdot \mathrm{pkt}_{\mathrm{j}}^{\mathrm{k}}-\frac{1}{\mathrm{~K}} \sum_{\mathrm{k}=1, \mathrm{k}^{\prime}=1}^{\mathrm{K}} \mathrm{pkt}_{\mathrm{i}}^{\mathrm{k}} \cdot \mathrm{pkt}_{\mathrm{j}}^{\mathrm{k}^{\prime}}\right)$

Route Metrics Evaluation fault detection

Cost lifetime of $i-t h$ light path $C(p i)$

Request holding period $R h$

Life time of $i-t h$ light path $p L i$

$$
R h-p L i \rightarrow \Delta t \text { (Adjustment constant) }
$$

Number hops in of $i-t$ th light path $H_{i}{ }^{n}$

$\varepsilon=10^{-5}$ (Exponential constant factor)

If new path found

$C(p i)=H_{i}{ }^{n} \times R h$

If $p L i \geq H_{i}{ }^{n} \quad$ then

$C(p i)=H_{i}^{n} \times \varepsilon$

If $p L i<H_{i}^{n} \quad$ then

$C(p i)=H_{i}^{n} *(\varepsilon+\Delta t)$

Return $G b[C(p i)]$

\section{Fitness Function Calculation}

The fitness function uses the available wavelengths of the designated path from source to destination node and the all-inclusive delay practiced by a burst. Fitness function can be calculated using the formula (3).

$\mathrm{F}(\mathrm{i})=\left[\gamma * \frac{(\operatorname{maxL}(\mathrm{s}, \mathrm{d})-\mathrm{L}(\mathrm{s}, \mathrm{d})}{\mathrm{L}(\mathrm{s}, \mathrm{d})} * \mathrm{Th}\right]+\left[(1-\gamma) *\left(1-\frac{\lambda_{\text {total }}-\lambda(\mathrm{s}, \mathrm{d})}{\lambda(\mathrm{s}, \mathrm{d})}\right) * \mathrm{Th}\right]$

The fitness function calculation is suitable only when the following constraint is satisfied.

$$
\lambda(s, d)>0 \& \lambda(s, d)<\lambda_{\text {total }}
$$

\section{Determination of Available Wavelength}

Wavelength assignment is resolved by using the Greedy algorithm from [1]. This is an upgrading algorithm which is run for a preferred number of iterations. From the literature, the greedy algorithm is utilized to minimize the number of wavelengths with respect to other wavelength assignment algorithm. The available total free bandwidth all over the route is taken into account in the selection of unconventional path. If it encounters no free wavelength for the estimated path, then immediately the fitness function neglects the routing path and the corresponding fitness value of the particle is symbolized as negative. $\max L(s, d)$ is the maximum route distance among all combination of source and destination pair and $L(s, d)$ is the route distance with reference to source ' $\mathrm{s}$ ' and destination ' $\mathrm{d}$ '. $\gamma[0,1]$ Is a design parameter. $\lambda_{\text {total }}$ is the total number of wavelengths. $\lambda(s, d)$ States the quantity of free wavelengths accessible through the route flanked by source's' and destination' $\mathrm{d}$ '.

\section{E. Significance of $\boldsymbol{\gamma}$-Design Parameter}

The value of $\gamma$ design parameter lies in the range of $[0,1]$ represent the blocking probability. The connection will be blocked, if there is non availability of link exist between source and destination. Probability of blocking is demarcated as the possibility that the connection could not be time-honored. The $\gamma$ is used to govern the influence of the path length and the quantity of available free wavelengths. The $\gamma$ value should be low for finding the better path intended for routing. This means that more free wavelengths are available in the particular path which also has very low traffic load. So, the $\gamma$ factor need to maintain dynamically that 
can offer extra weight to hop-length factor when the network load is stumpy and offer additional weight to 'number of free wavelengths' factor at restrained and high traffic loads.

\section{F. Delay Factor}

The delay of the packet is considered as another measure for the selection of optimal routing path in the optical network. The overall time variation between the estimated time and the actual time is known as delay. It is measured from the instant of its generation at the source node up to the delivery of aforementioned destination. This includes both propagation delay and queuing delay at the source and intermediate nodes. Generally, the bursts are assembled and transmitted to the destination. Every channel has an idle time in between the transmissions of consecutive bursts. This increases the delay time in optical networks. The average packet delay must be minimal for an efficient routing determination because the high-speed environment allows very little processing time. The delay factor can be acalculated using the formula (4) for each $j$ in light path $l p$. Then the determined delay factor value is Substituted in the equation (5). we can get Delay covariance between two routers $r_{i}, r_{j}$. This covariance value is used to find the fitness value of the particle for the aforementioned router. If delay covariance is high, when the burst traverses in the particular light path and then the algorithm assumes that there is fault in that particular path. Then we need to find an alternate path using the route metrics.

\section{G. Fault detection Evaluation Route Metrics}

Each established connection consumes the free wavelength and resources for a particular time in the dynamic environment. It is known as connection holding time. The light path assigned between the source and the destination has a random holding time for the transmission of bursts and then it is torn down. When there is fault in the particular path the holding time of a particular wavelength will be increased. If it exceeds with the lifetime of particular light path, we can assume that there is a fault. We compute the adjustment constant $\Delta t$ using holding time of particular wavelength needed by the request and Life time of $i_{t h}$ light path $p L i$ through the following formula (6).

The new determined routes have to gratify the succeeding constrictions.

$$
C(p i)=H_{i}^{n} \times R h
$$

We can assume the constraint satisfied route is suitable for the evaluation of next alternate path. Here there are two possibilities regarding $H_{i}{ }^{n}$ (Number of hops that occupies $i^{\text {th }}$ light path) for the determination of new path.

Case 1: If $p L i \geq H_{i}{ }^{n}$ then $\quad C(p i)=H_{i}{ }^{n} \times \varepsilon$

Case 2: If $p L i<H_{i}{ }^{n}$ then $C(p i)=H_{i}{ }^{n} *(\varepsilon+\Delta t)$

Where $\varepsilon=10^{-5}$ (Exponential constant factor)

Then finally the global best position value is updated through the Cost value of lifetime of $i^{\text {th }}$ light path $C(p i)$

\section{Performance ANAlysis}

On behalf of evaluating the performance of the proposed H-PSO algorithm for fault detection and survivability problem in footings of blocking probability and route calculation time, a simulation setup has been carry out with the assistance of Network Simulator (NS) -2 [23] by patching OBSs. It contemplates the mesh topology to simulate the NSF network with 14 nodes which is shown in fig 2. 


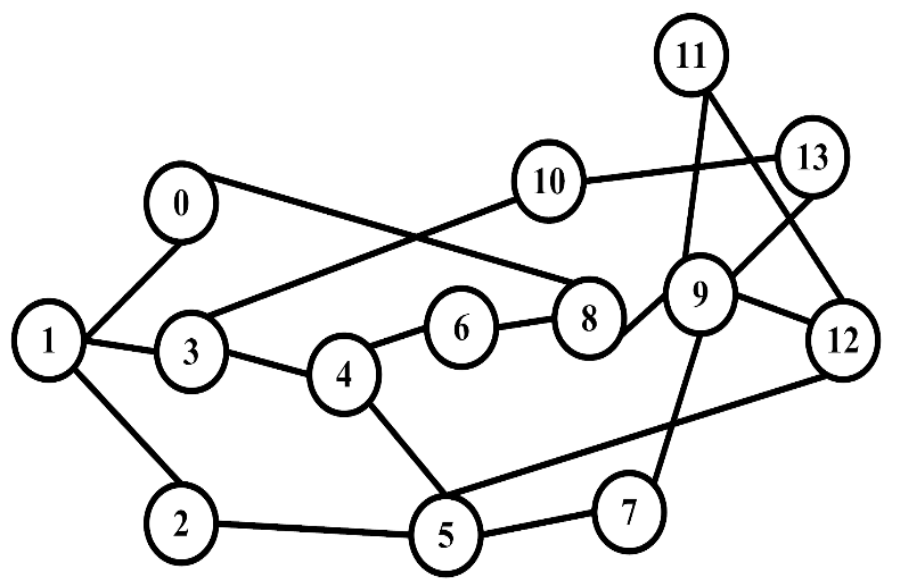

Figure2. NSFNET physical topology

\section{H. Assumptions}

1. Every single circuit connection use whole wavelength channel.

2. Each link possess similar amount of wavelengths.

3. Every single station possess groups of $\mathrm{K}$ transmitters and $\mathrm{K}$ receivers, where $\mathrm{K}$ is the quantity of wavelengths passed by fibers.

4. There is no queuing mechanism for the connection requests. If a connection request is refused/blocked, it is instantaneously rejected.

5. Indicators of link loads are equally autonomous and wavelength employments proceeding the links are equally self-governing. These are truly guesstimating, and the exactness of the model hinge on the network topology.

5. The Calls that could not be routed in the network are obstructed and lost.

6. Connection arrivals have Exponential distribution.

\section{Simulation Parameters}

\begin{tabular}{|l|c|}
\hline \multicolumn{1}{l}{ Simulation parameters } & \multicolumn{1}{c|}{ Values } \\
\hline Network topologies & NSFNET \\
\hline Link wavelength & 8 \\
\hline Packet Size (bytes) & 1250 \\
\hline Packet inter-arrival time & Exponential \\
\hline
\end{tabular}

Timer Threshold"

\begin{tabular}{|l|l|}
\hline Minimum & 0.001 \\
\hline Maximum & 0.006 \\
\hline Initial (t) & 0.004 \\
\hline
\end{tabular}

Burst Threshold (bytes)

\begin{tabular}{|l|c|}
\hline Minimum (m) & 1500 \\
\hline Maximum & 100000 \\
\hline Initial (l) & 60000 \\
\hline
\end{tabular}

Offered Load

\begin{tabular}{|l|c|}
\hline Minimum & 0.1 \\
\hline Maximum & 1 \\
\hline Increment & 0.1 \\
\hline
\end{tabular}


Take up the connection request arrivals are of exponential distribution. Contemplate the mesh topology by way of 14 nodes. Examine with assigned wavelengths in a link as 8 . As a result, the overall connection arrival rate $\left(\lambda_{\text {total }}\right)$ in the entire network is the product of complete quantity of nodes in the corresponding network and $\lambda_{\text {nodes }}$.

The total number of wavelengths in the optical network could be calculated using $\lambda_{\text {total }}=\alpha * \lambda_{\text {nodes }}$, where $\alpha$ is the total number nodes. We assume that the connection holding time is exponentially distributed with having mean value ' $\mathrm{T}$ ' secs.

As a result, the traffic load could be computed using Traffic load $=\lambda_{\text {total }} * T / \alpha *(\alpha-1)$ the initial setup of simulation experiment has been done according each node is supposed to have wavelength usage info of the entire network. No another routing is employed and not any further attempts are prepared on behalf of route re-computation. The connection establishment will be blocked when the selected route could not find a free wavelength. For every simulation round investigation, several traffic loads are employed. At very high traffic loads, established connections turn out to be flooded and the obtainability of free wavelengths befits very restricted as utmost of the wavelengths are by this time being used in the network. For that reason, PSO algorithm involves in finding further alternative routes in the case of encountering failure paths.

\section{Network Blocking Probability}

The graph is plotted to determine the blocking probability with respect to traffic load by implementing 8 and 16 wavelength variants. Fig 3 indicates the results obtained by heuristic PSO compared with other fault tolerance schemes. It shows that the blocking probability is expressively less than the former existing methodologies when the load increases. The main reason for this reduced blocking probability is effective use of resources such like availed wavelength and trunk utilization. All algorithm reacts similar process and provides same blocking probability performance at low traffic loads when we assign the wavelength capacity $8 /$ Link. i.e.

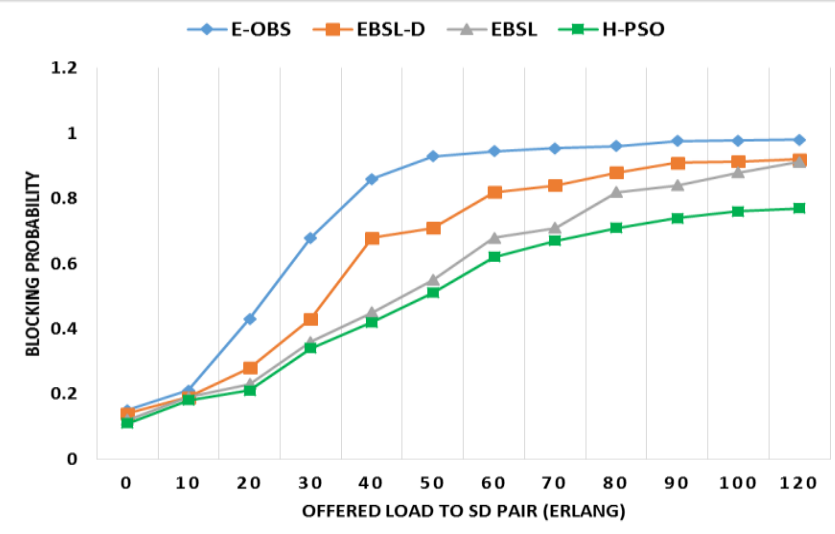

Figure 3. Offered Load Vs. Blocking Probability.

In the middle of the traffic loads of 0 and 10. The intention is, at low traffic loads, these algorithms discover free wavelengths for reserving so on route they pick. As soon as the traffic load is greater than before i.e., between 10 and 35, the H-PSO system indications represent a major performance enlargement in the context of blocking possibility. Conversely, while the traffic loads are enlarged above 40, the algorithm suffers without free wavelength. The blocking probability of our algorithm is 0.76 at maximum load. But the existing algorithms E-OBS, EBSL-D, EBSL provided 0.978, 0.914 and 0.88 respectively. Our algorithm achieved $14 \%$ reduced blocking probability than the existing best EBSL algorithm.

D. End-End Delay (ms) 


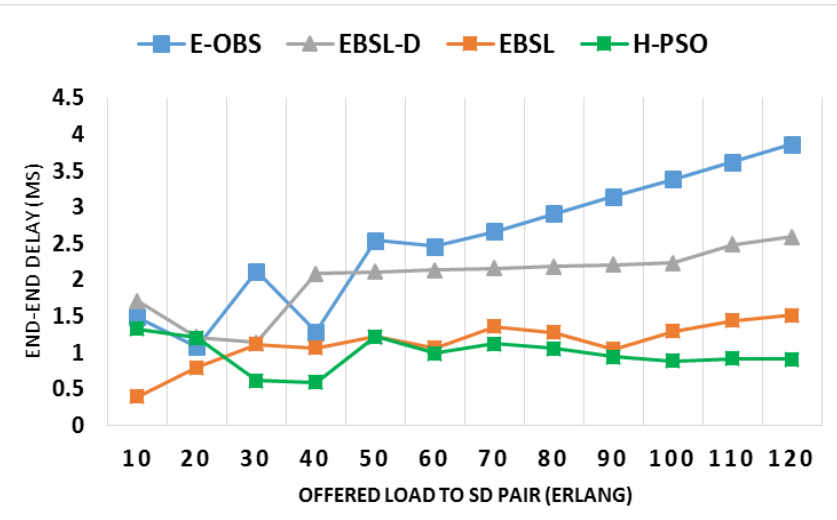

Figure 4. Offered Load Vs. End-End Delay (ms).

Fig 4 indicates the end-to-end delay performance regarding offered load. Generally, when the load increases the blocking probability increases and thus the end-End delay also raises. Delay is demarcated to be the time from a burst's appearance to the method in anticipation of the period that the burst finishes its transmission. This comprises both propagation delay and the required tuning time. The tuning time is defined as the required time for the adjustment of station channel in the destination side. Propagation time includes the required time of getting demanded bandwidth and transmission time. It can be also defined as the delay time until an Acknowledgement is received. The delay time of H-PSO is $0.9 \mathrm{~ms}$ at maximum offered load (120 Erlang). At the same time the other algorithms (E-OBS, EBSL-D, and EBSL) delay time is $3.86 \mathrm{~ms}, 2.59 \mathrm{~ms}$ and $1.50 \mathrm{~ms}$ respectively. The two factors (route distance and number of free available wavelengths) are standardized that involves in the computation of Fitness function. The benefit of exhausting such fitness function is that its diminutions the difference of average delay factor. So that the delay time of our proposed $\mathrm{H}-\mathrm{PSO}$ is reduced when comparing with other algorithms.

E. Throughput

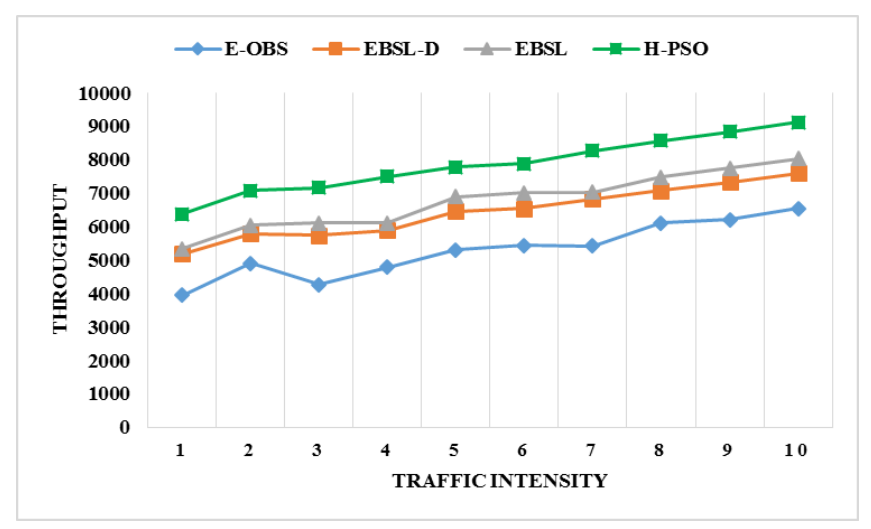

Figure 5. Throughput Vs Traffic Intensity

Fig 5 illustrates the throughput regarding the intensity of traffic.

Throughput is measured as the volume of data burst effectively directed to the endpoint terminus within the total simulation period. The optimal path can be analyzed and implemented by means of considering throughput as an objective parameter [26]. The mathematical formulation for throughput is expressed as

$$
\text { Throughput }=\frac{\text { Number of burst sent }(\text { bits })}{\text { Time period }(\text { seconds })}
$$

The Throughput of our H-PSO gradually increases due to heavy utilization of trunks. It is impervious to the dynamic bandwidth necessities of the network nodes and it is not effortlessly climbable in expressions of the amount of nodes. Thus, the QOS of our network is increased. At maximum load our algorithm provides the throughput value nearly 9124. But the other best existing algorithm EBSL offers the throughput only with 8031. Thus, our H-PSO achieves $11.97 \%$ high reliability in crucial traffic intensity. Our H-PSO dynamically 
identifies the faults and maintains the network reliability. Thus, the throughput increases when comparing with other algorithms.

\section{F. Burst Loss rate}

There is a chance of burst loss when the algorithm encounters heavy traffic. For an efficient algorithm the burst loss rate should be minimum for ensuring QOS parameters of the

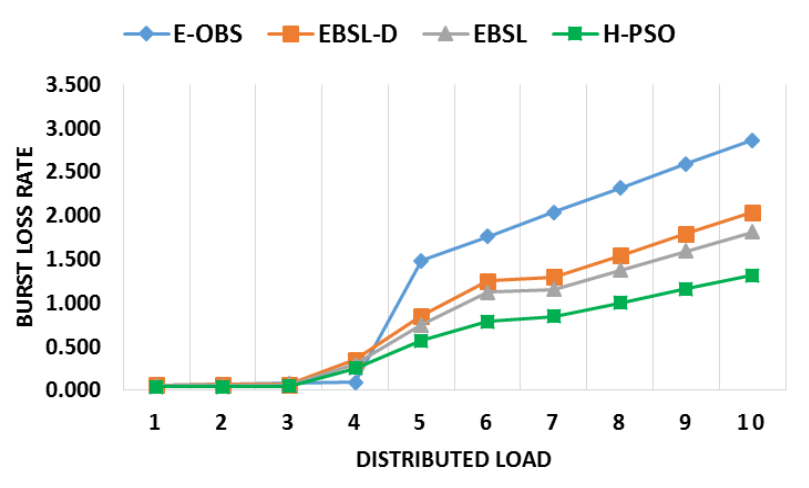

Figure 6. Burst Loss Rate Vs Traffic Intensity

network. Fig 6 shows that the proposed-PSO has very less burst loss rate when comparing with [24] other algorithms. The burst loss rate of H-PSO attained 1.32 in peak traffic intensity. At the same time the other algorithm's (E-OBS, EBSL-D, and EBSL) burst loss rate is 2.87, 2.04 and 1.82 respectively. Our H-PSO significantly reduces the burst loss rate upto 27\%. This indicates that our algorithm improves overall QOS parameters of the optical networks.

\section{CONCLUSION AND FUtURE WORK}

The performance of the submitted fault detection and network survivability algorithm based on Enhanced $\mathrm{H}$ PSO is analyzed in the optical network. The suggested fault detection algorithm can detect the failures and guarantee the determination of failures due to attacks in the optical networks. The damaged network component and their corresponding nodes were identified using the fitness function of H-PSO to each of the network Routing path. Enhanced H-PSO generates a preferred output to the fault circumstance when the trained input sets are evaluated. The new estimated alternate path is free from the faults and overloading which has optimal fitness value. We considered availed free wavelength and packet delay for the fitness calculation. Several network metric parameters viz., detection accuracy of the survivable network, miss detection ratio, recovery time and end-to-end delay has been considered. Our research work proved that the Enhanced H-PSO achieves fine on or after the fault discovery and new path determination, it is crucial to recommend a proper reclamation methodology that might competently recuperate from the understood faults. In future we need to evaluate hybrid combinations of evolutionary algorithms for optical network survivability that ensures the QOS parameters in light path communication.

\section{REFERENCES}

[1] N. Skorin-Kapov, J. Chen, and L. Wosinska, "A new approach to optical networks security: Attackaware routing and wavelength assignment," IEEE/ACM Transactions on Networking (TON), vol. 18, pp. 750-760, 2010.

[2] C. Mas and P. Thiran, "A review on fault location methods and their application to optical networks," 2001.

[3] H. Zeng, A. Vukovic, and C. Huang, "A novel end-to-end fault detection and localization protocol for wavelength-routed WDM networks," in Photonic Applications in Devices and Communication Systems, 2005, p. 59702D. 
[4] M. M. Rad, K. Fouli, H. A. Fathallah, L. A. Rusch, and M. Maier, "Passive optical network monitoring: challenges and requirements," IEEE Communications Magazine, vol. 49, 2011.

[5] A. Rodriguez, W. Fernández, and L. Ramírez, "RWA: Novel Heuristic Algorithm for Optical Networks with Dynamic Traffic," in Advanced Computer and Communication Engineering Technology, ed: Springer, 2016, pp. 1-10.

[6] Q. Li, B. Feng, L. Xie, P. Liang, H. Zhang, and T. Wang, "A Cross-Modality Learning Approach for Vessel Segmentation in Retinal Images," IEEE Trans. Med. Imaging, vol. 35, pp. 109-118, 2016.

[7] A. Agrawal, P. Sharma, V. Bhatia, and S. Prakash, "Survivability Improvement Against Earthquakes in Backbone Optical Networks Using Actual Seismic Zone Information," arXiv preprint arXiv:1703.02358, 2017.

[8] C. Wang, G. Shen, and S. K. Bose, "Distance adaptive dynamic routing and spectrum allocation in elastic optical networks with shared backup path protection," Journal of Lightwave Technology, vol. 33, pp. 2955-2964, 2015.

[9] A. Bhandari and J. Malhotra, "A Review on Network Survivability in Optical Networks," International Journal of Engineering Research and Applications, vol. 6, pp. 97-101, 2016.

[10] S. Hote, P. Ghodasara, T. Das, A. Kushwaha, S. Sharma, S. Bidkar, et al., "Developing and deploying a carrier-class sdn-centric network management system for a tier 1 service provider network," Journal of Optical Communications and Networking, vol. 9, pp. 711-729, 2017.

[11] K. Walkowiak, M. Klinkowski, B. Rabiega, and R. Goścień, "Routing and spectrum allocation algorithms for elastic optical networks with dedicated path protection," Optical Switching and Networking, vol. 13, pp. 63-75, 2014.

[12] X. Chen, S. Zhu, L. Jiang, and Z. Zhu, "On spectrum efficient failure-independent path protection pcycle design in elastic optical networks," Journal of Lightwave Technology, vol. 33, pp. 3719-3729, 2015.

[13] A. Giorgetti, F. Paolucci, F. Cugini, and P. Castoldi, "Fast restoration in SDN-based flexible optical networks," in Optical Fiber Communication Conference, 2014, p. Th3B. 2.

[14] A. Kushwaha, A. Gumaste, T. Das, S. Hote, and Y. Wen, "Flexible interconnection of scalable systems integrated using optical networks (FISSION) data-center-concepts and demonstration," Journal of Optical Communications and Networking, vol. 9, pp. 585-600, 2017.

[15] D. Amar, E. Le Rouzic, N. Brochier, and C. Lepers, "Multilayer restoration in elastic optical networks," in Optical Network Design and Modeling (ONDM), 2015 International Conference on, 2015, pp. 239-244.

[16] S. Lee, K. Sriram, H. Kim, and J. Song, "Contention-based limited deflection routing in OBS networks," in Global Telecommunications Conference, 2003. GLOBECOM'03. IEEE, 2003, pp. 2633-2637.

[17] Y.-C. Huang, Y. Yoshida, S. Ibrahim, R. Takahashi, A. Hiramatsu, and K.-i. Kitayama, "Bypassing Route Strategy for Optical Circuits in OPS-Based Data Center Networks," IEEE Photonics Journal, vol. 8, pp. 1-10, 2016.

[18] A. Hassan and C. Phillips, "Particle swarm optimization-based DRWA for wavelength continuous WDM optical networks using a novel fitness function," Artificial Intelligence Review, vol. 29, p. 305, 2008.

[19] H. C. Leung, C. S. Leung, E. W. Wong, and S. Li, "Extreme learning machine for estimating blocking probability of bufferless OBS/OPS networks," Journal of Optical Communications and Networking, vol. 9, pp. 682-692, 2017.

[20] U. R. Bhatt, T. Sarsodia, R. Upadhyay, and R. Sharan, "Implementation of Ant Colony Optimization Algorithm for Survivable optical network," 2016.

[21] Z. Wang, H. Xing, T. Li, Y. Yang, R. Qu, and Y. Pan, "A modified ant colony optimization algorithm for network coding resource minimization," IEEE Transactions on Evolutionary Computation, vol. 20, pp. 325-342, 2016. 
[22] G. S. Pavani, A. de França Queiroz, and J. C. Pellegrini, "Analysis of ant colony optimization-based routing in optical networks in the presence of byzantine failures," Information Sciences, vol. 340, pp. 27-40, 2016.

[23] B. Yang, L. Wang, L. Ning, and K. Zeng, "Effects of pilot injection timing on the combustion noise and particle emissions of a diesel/natural gas dual-fuel engine at low load," Applied Thermal Engineering, vol. 102, pp. 822-828, 2016.

[24] L. Shuo, "Analysis and Synthesis of Optical Burst Switched Networks," 2014.

[25] J.Kumarnath and K.Batri, " A BAT algorithm based Enhancement of physical layer security in a Multi Domain-Elastic Optical Network, "International Journal of Pure and Applied Mathematics,vol.119,pg 2519-2525, 2018.

[26] J.Kumarnath J and Dr. Batri K., A Novel parametric Analysis of the performance dynamicity of Optical Network.Advances in Natural and Applied Sciences. 11(9); pp: 166-173,2017

[27] Zhuqin Chu, Hui Li, Huaxi Gu, Xiaochun Ye, Wavelength assignment method based on ACO to reduce crosstalk for ring-based optical Network-on-Chip. Microprocessors and Microsystems, Vol 71.https://doi.org/10.1016/j.micpro.2019.102849, 2019.

[28] Issam Damaj, Mohamed Elshafei, Mohammed El-Abd, Mehmet Emin Aydin "An analytical framework for high-speed hardware particle swarm optimization," Microprocessors and Microsystems, vol. 72, https://doi.org/10.1016/j.micpro.2019.102949,2020.

[29] Shi Peilong, Zhao Xuan, Liu Wentao, Zhou Wenhui, Yu Qiang and Zhang Shuo, "Composite Control for Path Tracking of an Intelligent Vehicle Base on Particle Swarm Optimization and Bezier Curve," Dynamic Systems and Applications, vol.29, pp.2635 - 2655,2020.

[30] N. K. Priyadharsini and D. Chitra, "A Machine Learning and Swarm Intelligence Based Approaches for Anomaly Detection in Extremely Crowded Scenes,"Dynamic Systems and Applications, vol.30, pp. $1250-1272,2021$. 